\title{
Molecular hydrogen: an overview of its neurobiological effects and therapeutic potential for bipolar disorder and schizophrenia
}

\author{
Ahmad Ghanizadeh ${ }^{1,2^{*}}$ and Michael Berk ${ }^{3,4,5}$
}

\begin{abstract}
Hydrogen gas is a bioactive molecule that has a diversity of effects, including anti-apoptotic, anti-inflammatory and anti-oxidative properties; these overlap with the process of neuroprogression in major psychiatric disorders. Specifically, both bipolar disorder and schizophrenia are associated with increased oxidative and inflammatory stress. Moreover, lithium which is commonly administered for treating bipolar disorder has effects on oxidative stress and apoptotic pathways, as do valproate and some atypical antipsychotics for treating schizophrenia. Molecular hydrogen has been studied pre-clinically in animal models for the treatment of some medical conditions including hypoxia and neurodegenerative disorders, and there are intriguing clinical findings in neurological disorders including Parkinson's disease. Therefore, it is hypothesized that administration of hydrogen molecule may have potential as a novel therapy for bipolar disorder, schizophrenia, and other concurrent disorders characterized by oxidative, inflammatory and apoptotic dysregulation.
\end{abstract}

\section{Introduction}

Normally, there is a balance between oxidant and antioxidant systems in body. When there is an imbalance between anti-oxidative defenses and prevailing oxidative stress, reactive oxidative species are increased leading to inflammation and oxidative damage marked by protein carbonylation, DNA damage [1,2] and lipid peroxidation. These biomarkers are documented in all illness phases, but appear more pronounced in episodes of acute illness, particularly mania [3].

Bipolar mood disorder is a relatively common neuropsychiatric disorder with an estimated prevalence of $1 \%$ to $2 \%$ [4], and a high burden of disease [4]. There is a high rate of medical comorbidity including diabetes and the metabolic syndrome [5], cardiovascular morbidity [6], and obesity [7], all of which are associated with inflammatory changes and oxidative stress. These comorbidities lead to a novel hypothesis that bipolar disorder is a part of a multi-system inflammatory process [8].

\footnotetext{
* Correspondence: ghanizad@sina.tums.ac.ir

${ }^{1}$ Research Center for Psychiatry and Behavioral Sciences, Shiraz University of Medical, Sciences, Hafez Hospital, Shiraz, Iran

${ }^{2}$ Department of Psychiatry, Hafez Hospital, School of Medicine, Shiraz

University of Medical Sciences, Shiraz, Iran

Full list of author information is available at the end of the article
}

Additionally, schizophrenia has a point prevalence of $3.59 / 1000$ in general population [9]. The mean Canadian rates is 25.9 (S.D. $=10.5)$ per 100,000 [10]. Schizophrenia is also comorbid with medical conditions such as diabetes (adjusted OR: 2.11 (1.36 to 3.28) [11], overweight or obesity (44\%) [12] and cardiovascular events [13]. Schizophrenia as well as bipolar disorder are part of a multisystem inflammatory processes [14] and anti-inflammatory therapy for treating schizophrenia is suggested [15].

\section{Mitochondria and bipolar mood disorder}

Mitochondria are necessary for the generation of energy and synaptic signaling. Mitochondrial dysfunction appears to be involved in the pathophysiology of bipolar disorder [16]. The prevalence of bipolar disorder in patients with mitochondrial cytopathies is higher than among healthy controls [17]. Reduction in complex one activity of the mitochondrial electron transport chain is documented [18], and there are intriguing preliminary electron microscopy reports of mitochondrial ultrastructural changes in the disorder, particularly abnormal peripheral clustering of mitochondrial in the cytosol [19]. Mitochondrial DNA deletions are increased [20]. Mitochondrial dysfunction has thus been hypothesizes as a

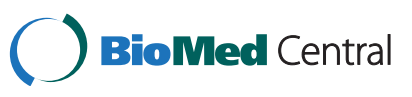


molecular basis of bipolar disorder [21]. It is suggested that novel therapeutic agents for treating bipolar mood disorder should target mitochondrial function [16].

\section{Bipolar disorder and oxidative stress}

The generation of oxidatively generated free radicals is core to life, and is normally tightly controlled. However, there is now increasing data on the presence and impact of oxidative stress among diverse psychiatric disorders including bipolar disorder [22], schizophrenia, and autism [23,24]. Reactive oxygen species (ROS) production and metabolism appears to play an important role in the pathophysiology of bipolar disorder $[22,25,26]$. Not only are both total glutathione and reduced glutathione levels, which are core parts of the intrinsic anti-oxidative system decreased in bipolar disorder, but antioxidant enzyme activities are also impaired [22]. Levels of key redox enzymes including SOD and catalase have been reported to be lower in patients with bipolar disorder than in matched controls [27]. Nitrous oxide (NO) is implicated in the generation of psychotic symptoms such as delusions in bipolar disorder [28]. A meta-analysis reported that the level of $\mathrm{NO}$ appears to be significantly increased in bipolar disorder [29]. Moreover, the level of thiobarbituric acidic reactive substances (TBARS), a marker of lipid peroxidation through reaction between free radicals and lipid structures, is increased during manic episodes and remission [30,31]. This finding suggests that oxidative damage to lipid structures is probably continued during the course of bipolar disorder.

\section{Mechanism of action of established therapies}

Lithium is arguably the most effective medication for the prevention of long-term relapse in bipolar mood disorder [32,33]. Lithium's effects extend beyond its mode of action of monoamine receptors [34]. Chronic treatment with lithium leads to mitochondrial proteins phosphorylation in the rat prefrontal cortex [34]. Lithium also has effects through its impact on inflammation and by the prevention of oxidative stress and cytokine changes [35]. These anti-inflammatory and antioxidative effects are potentially neuroprotective [36,37]. Lithium increases mitochondrial respiratory chain enzyme activities, particularly complex 1 , that may be linked to its therapeutic efficacy [38]. Lithium increases mitochondrial energy production [38]. Decreased $\mathrm{Na}(+)-\mathrm{K}$ $(+)$-ATPase activity and increased lipid peroxidation in that are seen in bipolar disorder may improve following lithium administration [39]. Long term lithium administration enhances anti-oxidative defenses in bipolar disorder [40] as well as in healthy individuals [41]. $\mathrm{N}$-acetyl-cysteine (NAC), a glutathione based redox modulator, anti-inflammatory agent and mitochondrial modulator [42] decreases symptoms of depression and mania
[43] in bipolar disorder [44]. However, established agents have tolerability issues and efficacy limitations, therefore more novel therapeutic approached are needed.

\section{Mitochondria and schizophrenia}

Mitochondrial dysfunction in schizophrenia is frequently reported [45]. Moreover, mitochondrial disorders can present with psychosis [46]. mtDNA plays a role in the neurobiology of schizophrenia [47]. Mitochondrial gene expression is changed in schizophrenia [48]. The numbers of mitochondria in schizophrenia is reduced compared to normal controls [49]. This change in mitochondria may be associated with differential responsiveness to treatment [50]. However, it is not clear whether this number is adaptive or an etiological link to this disorder [51]. Moreover, mitochondrial energy production is impaired in autism [52].

\section{Schizophrenia and oxidative stress}

The role oxidative stress in the neurobiology of schizophrenia is a promising target in order to provide new therapeutic interventions [53]. This is grounded on data that the antioxidant defense system is impaired in schizophrenia [54]. In comparison to normal controls, the activities of superoxide dismutase (SOD), glutathione peroxidase (GSH-Px) are decreased while the levels of MDA are increased chronic schizophrenia [55]. Reduced cellular respiration and complex I abnormalities in schizophrenia are a possible endophenotypic biomarker for schizophrenia [56]. Furthermore, the severity of neurological soft signs in patients with schizophrenia is associated with the level of decreased superoxide dismutase activity [57]. Treatment refractory schizophrenia is associated with higher lipid peroxidation [58].

\section{Mechanism of action of antipsychotics}

The increase of plasma lipid peroxidation in schizophrenia is not due to second-generation antipsychotics [59]. But both typical and atypical antipsychotic medication partially normalize abnormal free radical metabolism in schizophrenia [60]. Long term treatments with both typical and atypical antipsychotics have effects on antioxidant enzymes and lipid peroxidation in schizophrenia [55]. Supplementation of vitamin $C$ with atypical decreases oxidative stress in schizophrenia [61]. $\mathrm{N}$ actylcysteine, a redox modulator and glutamate modulator, reduces core symptoms and akathisia in schizophrenia, providing a preliminary proof of concept for the role of oxidative pathways [62].

\section{Pharmacokinetics of Hydrogen}

Molecular hydrogen is an odourless and tasteless gas that has the ability to rapidly diffuse through lipid membranes and enter the cell, where it easily penetrates 
organelles such as mitochondria as well as the nucleus. It is inert at room temperature and in the absence of catalysts. It additionally easily crosses the blood brain barrier, which facilitates access to the target organs and subcellular components. Its adverse event profile is reportedly benign $[2,63]$. Physiologically, hydrogen is produced by intestinal microbiota from fermentation of complex carbohydrates. Arterial blood contains higher levels of hydrogen that tissue, suggesting some uptake and utilization of hydrogen in tissues. Hydrogen reacts with free hyrodoxyl radicals but does not appear to react to other reactive oxygen species. This is a theoretical advantage, as low levels of these radicals have physiologically relevant signaling effects. It protects against secondary oxidative damage to the brain in a variety of models by reacting with hydroxyl radicals [64]. Hydrogen is potentially available as a medical gas, hydrogen enriched water, taking a hydrogen bath, injecting hydrogen saline, hydrogen saline eye drops, and augmenting bacterial production of intestinal hydrogen. It penetrates glass but not aluminum containers.

\section{Hydrogen and oxidative stress and inflammation}

Anti-inflammatory effects of hydrogen molecule have been reported in both animal and human models. Hydrogen-rich saline decreases the levels of cytokines IL-4, IL-5, IL-13 and TNF- $\alpha$ in bronchoalveolar lavage fluid [65]. Its effects on tumor necrosis factor alpha, interleukin (IL)-1 $\beta$ and IL-6 levels is hypothesized as the reason for its protective role against UVB radiation [66]. Hydrogen molecule also stops TNF $\alpha$-induced activation of the $\mathrm{NF}_{\kappa} \mathrm{B}$ pathway [67]. Hydrogen released by intestinal microbiota, such a hydrogen producing strain of E. coli can suppress inflammation [68]. Taken together, these findings are germane to bipolar disorder as current evidences implicates many interleukins in the neurobiology of bipolar disorder [69].

\section{Preclinical findings}

Inhalation of hydrogen decreased acute lung inflammation in an animal model of acute lung injury [70,71]. Hydrogen enriched water decreased the production of reactive oxygen species in the rat kidney [72]. It protects mitochondria and nuclear DNA from hydroxyl radicals, preventing the decline in mitochondrial membrane potential after antimycin treatment. It has potential in preventing ionizing radiation induced damage, as hydroxyl radicals are the major vehicle for secondary damage. Hydrogen reduces infarction size in a model of middle cerebral artery occlusion and reduced oxidative stress markers [73]. In asphyxiated newborn piglets, it is similarly neuroprotective and preserves cerebrovascular reactivity [74].

\section{Clinical findings}

Hydrogen is hypothesized as a potential therapy for different oxidative stress related diseases such as Parkinson's disease; ischemia/reperfusion of spinal cord, heart, lung, liver, kidney, and intestine; transplantation of lung, heart, kidney, and intestine [75], and autism [76,77]. Adequate hydration with hydrogen-rich water decreases blood lactate levels and improved function of muscle in athletes [78].

Among participants with type 2 diabetes, hydrogen reduced levels of oxidative stress markers [68], and similar findings are reported in the metabolic syndrome [79]. In Parkinson's disease, increased oxidative stress indexed by elevated lipid peroxidation and decreased reduced glutathione levels in the substantia nigra are part of the known pathogenesis of PD. Hydrogen water also prevents a rat model of Parkinson's disease [80] and increases survival after cerebral ischemia/reperfusion [81,82]. It down-regulates 4-hydroxy-2-nonenal, a marker of oxidative stress in dopaminergic neurons within the substantia nigra of animal models of Parkinson's disease. A preclinical study failed to find any association between response to hydrogen and dose [37]. In a pilot placebo controlled, randomized, double-blind, parallel-group design $(\mathrm{N}=18)$, the efficacy of $1000 \mathrm{ml}$ molecular hydrogen daily was investigated in patients taking levodopa. In the hydrogen group, there was a reduction in Total Unified Parkinson's Disease Rating Scale scores, whereas the placebo group deteriorated [83]. Signal modulating activities of hydrogen may play a role in exerting a protective effect against Parkinson's [79].

It is not fully understood how hydrogen plays its putative antioxidative or anti-inflammatory role. However, it may regulate particular metalloproteins [84]. Moreover, hydrogen reduces the production of peroxynitrite derived from nitric oxide [85]. In patients with rheumatoid arthritis, drinking of water enriched with hydrogen was shown to decreases oxidative stress through scavenging hydroxyl radical and also decreases clinical symptoms after 4 weeks [86]. Moreover, hydrogen-enriched water improves quality of life of patients with liver cancer after radiation exposure [87].

Intravenous administration of $500 \mathrm{ml}$ of $\mathrm{H} 2$ enriched fluid has also been examined and it decreased the symptoms of 4 patients with acute erythematous skin diseases with fever and/or pain [88]. A randomized controlled trial indicated that hydrogen-enriched water decreased mitochondrial dysfunction and inflammation in patients with mitochondrial myopathies [89].

Caveats are however necessary, inasmuch as orally administered hydrogen-enriched water may not have enough molecular hydrogen to adequately scavenge hydroxyl radicals. Secondly, the dwell time of hydrogen in the body may be too short to scavenge substantial quantities of 
continuously generated hydroxyl radicals [90]. In addition, the optimal frequency, dosage, and its method of administration for different diseases are not studied [75].

\section{Conclusions}

Hydrogen gas has a number of biological properties that make it an appealing candidate agent for a diversity of disorders sharing inflammatory, oxidative and apoptotic mechanisms. However, a number of caveats are necessary. Administration is complex, aggravated by its very short biological half-life and low saturation point of $0,8 \mathrm{mM}$. Furthermore, the low dose used in many studies $(0.04-0.08 \mathrm{mM})$ is shadowed by the fact that intestinal microbiota produce up to $1 \mathrm{~L}$ a day of hydrogen [91]. There is currently very a limited understanding of the pathways and processes impacted by hydrogen. Greater preclinical data is required to elucidate the biological mechanisms of hydrogen. It however is true that many agents are in widespread use despite poor understanding of their mechanisms of action, particularly in the neurosciences. This therefore does not preclude use, which is therefore far more contingent on safety issues. Given that hydrogen does seem to have a benign safety profile, albeit based on very limited data, moving to clinical studies appears warranted. We additionally need a more complete understanding of hydrogen's dosage, mode of administration, pharmacokinetics, biology and toxicity of hydrogen to facilitate clinical application. $\mathrm{Ne}$ vertheless, there is much that is appealing about this avenue, and it merits greater investment given the paucity of therapeutic options and their limitations.

\section{Competing interests}

AG has no related conflict of interest to be declared. MB has received Grant/ Research Support from the $\mathrm{NIH}$, Cooperative Research Centre, Simons Autism Foundation, Cancer Council of Victoria, Stanley Medical Research Foundation, MBF, NHMRC, Beyond Blue, Rotary Health, Geelong Medical Research Foundation, Bristol Myers Squibb, Eli Lilly, Glaxo SmithKline, Organon, Novartis, Mayne Pharma and Servier, has been a speaker for Astra Zeneca, Bristol Myers Squibb, Eli Lilly, Glaxo SmithKline, Janssen Cilag, Lundbeck, Merck, Pfizer, Sanofi Synthelabo, Servier, Solvay and Wyeth, and served as a consultant to Astra Zeneca, Bristol Myers Squibb, Eli Lilly, Glaxo SmithKline, Janssen Cilag, Lundbeck Merck and Servier, and is a co-inventor of two provisional patents regarding the use of NAC and related compounds for psychiatric indications, which, while assigned to the Mental Health Research Institute, could lead to personal remuneration upon a commercialization event.

\section{Authors' contributions}

AG was responsible for the initial conception and drafting of the manuscript. $A G$ and $M B$ revised the preliminary draft of the manuscript. Both authors read and approved the final manuscript.

\section{Author details}

'Research Center for Psychiatry and Behavioral Sciences, Shiraz University of Medical, Sciences, Hafez Hospital, Shiraz, Iran. ${ }^{2}$ Department of Psychiatry, Hafez Hospital, School of Medicine, Shiraz University of Medical Sciences, Shiraz, Iran. ${ }^{3}$ School of Medicine, Deakin University, Melbourne, Australia. ${ }^{4}$ The Florey Institute of Neuroscience and Mental Health, Orygen Research Centre, University of Melbourne, 234 St Kilda Rd, Southbank VIC 3006, Australia. ${ }^{5}$ The Department of Psychiatry, University of Melbourne, $234 \mathrm{St}$ Kilda Rd, Southbank, VIC 3006, Australia.
Received: 13 December 2012 Accepted: 30 May 2013

Published: 6 June 2013

\section{References}

1. Andreazza AC, et al: Specific subcellular changes in oxidative stress in prefrontal cortex from patients with bipolar disorder. J Neurochem 2013 [Epub ahead of print].

2. Soeiro-de-Souza MG, et al: Number of manic episodes is associated with elevated DNA oxidation in bipolar I disorder. Int I Neuropsychopharmacol 2013:1-8 [Epub ahead of print].

3. Frey BN, et al: Biomarkers in bipolar disorder: a positional paper from the International Society for Bipolar Disorders Biomarkers Task Force. Aust N Z J Psychiatry 2013, 47(4):321-32.

4. Fagiolini A, et al: Prevalence, chronicity, burden and borders of bipolar disorder. J Affect Disord 2013, 148(2-3):161-9.

5. Bai YM, et al: Risk of developing diabetes mellitus and hyperlipidemia among patients with bipolar disorder, major depressive disorder, and schizophrenia: A 10-year nationwide population-based prospective cohort study. J Affect Disord 2013 [Epub ahead of print].

6. Callaghan RC, Khizar A: The incidence of cardiovascular morbidity among patients with bipolar disorder: a population-based longitudinal study in Ontario. Canada. J Affect Disord 2010, 122(1-2):118-23.

7. Gurpegui $M$, et al: Overweight and obesity in patients with bipolar disorder or schizophrenia compared with a non-psychiatric sample. Prog Neuropsychopharmacol Biol Psychiatry 2012, 37(1):169-75.

8. Leboyer $\mathrm{M}$, et al: Can bipolar disorder be viewed as a multi-system inflammatory disease? J Affect Disord 2012, 141(1):1-10.

9. Shivashankar S, et al: Has the prevalence, clinical presentation and social functioning of schizophrenia changed over the last 25 years? Nithsdale schizophrenia survey revisited. Schizophr Res 2013, 146(1-3):349-56.

10. Dealberto MJ: Are the rates of schizophrenia unusually high in Canada? A comparison of Canadian and international data. Psychiatry Res 2013 [Epub ahead of print].

11. Carney CP, Jones LE: Medical comorbidity in women and men with bipolar disorders: a population-based controlled study. Psychosom Med 2006, 68(5):684-91

12. Guo $X$, et al: The relationship between obesity and neurocognitive function in Chinese patients with schizophrenia. BMC Psychiatry 2013, 13:109.

13. Protopopova $D$, et al: The prevalence of cardiometabolic risk factors and the ten-year risk of fatal cardiovascular events in patients with schizophrenia and related psychotic disorders. Psychiatr Danub 2012, 24(3):307-13.

14. Falcone $T$, et al: Does systemic inflammation play a role in pediatric psychosis. Clin Schizophr Relat Psychoses 2013:1-43 [Epub ahead of print].

15. Muller $\mathrm{N}$, et al: Anti-inflammatory treatment in schizophrenia. Prog Neuropsychopharmacol Biol Psychiatry 2013, 42:146-53.

16. Kato T: The role of mitochondrial dysfunction in bipolar disorder. Drug News Perspect 2006, 19(10):597-602.

17. Fattal $\mathrm{O}$, et al: Psychiatric comorbidity in 36 adults with mitochondrial cytopathies. CNS Spectr 2007, 12(6):429-38.

18. Scola G, et al: A fresh look at complex I in microarray data: clues to understanding disease-specific mitochondrial alterations in bipolar disorder. Biol Psychiatry 2013, 73(2):e4-5.

19. Cataldo AM, et al: Abnormalities in mitochondrial structure in cells from patients with bipolar disorder. Am J Pathol 2010, 177(2):575-85.

20. Kato T, et al: Increased levels of a mitochondrial DNA deletion in the brain of patients with bipolar disorder. Biol Psychiatry 1997, 42(10):871-5.

21. Kato T: Mitochondrial dysfunction as the molecular basis of bipolar disorder: therapeutic implications. CNS Drugs 2007, 21(1):1-11.

22. Raffa M, et al: Reduced antioxidant defense systems in schizophrenia and bipolar I disorder. Prog Neuropsychopharmacol Biol Psychiatry 2012, 39(2):371-5

23. Ghanizadeh A, et al: Glutathione-related factors and oxidative stress in autism, a review. Curr Med Chem 2012, 19(23):4000-5.

24. Ghanizadeh A: Gold nanoparticles and lipoic acid as a novel antiinflammatory treatment for autism, a hypothesis. Journal of Medical Hypotheses and Ideas 2012, 6(1):40-43.

25. Kulak A, et al: Redox dysregulation in the pathophysiology of Schizophrenia and bipolar disorder: insights from animal models. Antioxid Redox Signal 2012, 18(12):1428-43. 
26. Steckert AV, et al: Role of oxidative stress in the pathophysiology of bipolar disorder. Neurochem Res 2010, 35(9):1295-301.

27. Ranjekar PK, et al: Decreased antioxidant enzymes and membrane essential polyunsaturated fatty acids in schizophrenic and bipolar mood disorder patients. Psychiatry Res 2003, 121(2):109-22

28. Gergerlioglu HS, et al: Changes in nitric oxide level and superoxide dismutase activity during antimanic treatment. Prog Neuropsychopharmacol Biol Psychiatry 2007, 31(3):697-702.

29. Andreazza $A C$, et al: Oxidative stress markers in bipolar disorder: a meta-analysis. J Affect Disord 2008, 111(2-3):135-44.

30. Andreazza AC, et al: Serum S100B and antioxidant enzymes in bipolar patients. J Psychiatr Res 2007, 41(6):523-9.

31. Frey BN, et al: Increased oxidative stress and DNA damage in bipolar disorder: a twin-case report. Prog Neuropsychopharmacol Biol Psychiatry 2007, 31(1):283-5.

32. Geddes JR, Miklowitz DJ: Treatment of bipolar disorder. Lancet 2013, 381(9878):1672-82.

33. Malhi GS, et al: Potential mechanisms of action of lithium in bipolar disorder. Current understanding. CNS Drugs 2013, 27(2):135-53.

34. Corena-McLeod M, et al: New model of action for mood stabilizers: phosphoproteome from rat pre-frontal cortex synaptoneurosomal preparations. PLoS One 2013, 8(5):e52147.

35. Albayrak A, et al: Protective effects of lithium: A new look at an old drug with potential antioxidative and anti-inflammatory effects in an animal model of sepsis. Int Immunopharmacol 2013, 16(1):35-40.

36. Chiu CT, et al: Therapeutic potential of mood stabilizers lithium and valproic acid: beyond bipolar disorder. Pharmacol Rev 2013, 65(1):105-42.

37. Yu F, et al: Lithium ameliorates neurodegeneration, suppresses neuroinflammation, and improves behavioral performance in a mouse model of traumatic brain injury. J Neurotrauma 2012, 29(2):362-74.

38. Maurer IC, Schippel P, Volz HP: Lithium-induced enhancement of mitochondrial oxidative phosphorylation in human brain tissue. Bipolar Disord 2009, 11(5):515-22.

39. Banerjee $\mathrm{U}$, et al: Effects of lithium therapy on $\mathrm{Na}+-\mathrm{K}+-$ ATPase activity and lipid peroxidation in bipolar disorder. Prog Neuropsychopharmacol Biol Psychiatry 2012, 37(1):56-61.

40. Arraf $Z$, et al: Lithium and oxidative stress lessons from the MPTP model of Parkinson's disease. Neurosci Lett 2012, 516(1):57-61.

41. Khairova $R$, et al: Effects of lithium on oxidative stress parameters in healthy subjects. Mol Med Report 2012, 5(3):680-2.

42. Samuni $Y$, et al: The chemistry and biological activities of $\mathrm{N}$ acetylcysteine. Biochim Biophys Acta 2013, 1830(8):4117-4129.

43. Magalhaes PV, et al: A preliminary investigation on the efficacy of $\mathrm{N}$-acetyl cysteine for mania or hypomania. Aust N Z J Psychiatry 2013, 47(6):564-8.

44. Berk $\mathrm{M}$, et al: $\mathrm{N}$-acetyl cysteine for depressive symptoms in bipola disorder-a double-blind randomized placebo-controlled trial. Biol Psychiatry 2008, 64(6):468-75.

45. Park C, Park SK: Molecular links between mitochondrial dysfunctions and schizophrenia. Mol Cells 2012, 33(2):105-10.

46. Anglin RE, et al: The psychiatric manifestations of mitochondrial disorders: a case and review of the literature. J Clin Psychiatry 2012, 73(4):506-12.

47. Verge B, et al: Mitochondrial DNA (mtDNA) and schizophrenia. Eur Psychiatry 2011, 26(1):45-56.

48. Whatley SA, Curti D, Marchbanks RM: Mitochondrial involvement in schizophrenia and other functional psychoses. Neurochem Res 1996, 21(9):995-1004.

49. Somerville SM, Conley RR, Roberts RC: Striatal mitochondria in subjects with chronic undifferentiated vs. chronic paranoid schizophrenia. Synapse 2012, 66(1):29-41.

50. Somerville SM, et al: Mitochondria in the striatum of subjects with schizophrenia: relationship to treatment response. Synapse 2011, 65(3):215-24.

51. Somerville SM, Conley RR, Roberts RC: Mitochondria in the striatum of subjects with schizophrenia. World J Biol Psychiatry 2011, 12(1):48-56.

52. Rezin GT, et al: Mitochondrial dysfunction and psychiatric disorders. Neurochem Res 2009, 34(6):1021-9.

53. Boskovic M, et al: Oxidative stress in schizophrenia. Curr Neuropharmacol 2011, 9(2):301-12.

54. Virit $O$, et al: $A$ defect in the antioxidant defense system in schizophrenia. Neuropsychobiology 2009, 60(2):87-93.
55. Zhang $X Y$, et al: Antioxidant enzymes and lipid peroxidation in different forms of schizophrenia treated with typical and atypical antipsychotics. Schizophr Res 2006, 81(2-3):291-300.

56. Rosenfeld $M$, et al: Perturbation in mitochondrial network dynamics and in complex I dependent cellular respiration in schizophrenia. Biol Psychiatry 2011, 69(10):980-8.

57. Raffa $M$, et al: The reduction of superoxide dismutase activity is associated with the severity of neurological soft signs in patients with schizophrenia. Prog Neuropsychopharmacol Biol Psychiatry 2012, 39(1):52-6.

58. Medina-Hernandez $V$, et al: Increased lipid peroxidation and neuron specific enolase in treatment refractory schizophrenics. J Psychiatr Res 2007, 41(8):652-8.

59. Dietrich-Muszalska A, Kontek B: Lipid peroxidation in patients with schizophrenia. Psychiatry Clin Neurosci 2010, 64(5):469-75.

60. Zhang $X Y$, et al: Effects of risperidone and haloperidol on superoxide dismutase and nitric oxide in schizophrenia. Neuropharmacology 2012, 62(5-6):1928-34

61. Dakhale $\mathrm{GN}$, et al: Supplementation of vitamin $\mathrm{C}$ with atypical antipsychotics reduces oxidative stress and improves the outcome of schizophrenia. Psychopharmacology (Berl) 2005, 182(4):494-8.

62. Berk $\mathrm{M}$, et al: $\mathrm{N}$-acetyl cysteine as a glutathione precursor for schizophrenia-a double-blind, randomized, placebo-controlled trial. Biol Psychiatry 2008, 64(5):361-8.

63. Dixon BJ, Tang J, Zhang JH: The evolution of molecular hydrogen: a noteworthy potential therapy with clinical significance. Med Gas Res 2013, 3(1):10.

64. Ohsawa l, et al: Hydrogen acts as a therapeutic antioxidant by selectively reducing cytotoxic oxygen radicals. Nat Med 2007, 13(6):688-94.

65. Xiao $M$, et al: Hydrogen-rich saline reduces airway remodeling via inactivation of NF-kappaB in a murine model of asthma. Eur Rev Med Pharmacol Sci 2013, 17(8):1033-43.

66. Guo $Z$, et al: Hydrogen-rich saline protects against ultraviolet $B$ radiation injury in rats. J Biomed Res 2012, 26(5):365-71.

67. Cai WW, et al: Treatment with hydrogen molecule alleviates TNFalphainduced cell injury in osteoblast. Mol Cell Biochem 2013, 373(1-2):1-9.

68. Kajiya $M$, et al: Hydrogen from intestinal bacteria is protective for Concanavalin A-induced hepatitis. Biochem Biophys Res Commun 2009, 386(2):316-21.

69. Modabbernia A, et al: Cytokine Alterations in Bipolar Disorder: a Meta-Analysis of 30 Studies. Biol Psychiatry 2013 [Epub ahead of print].

70. Faller $S$, et al: Inhaled hydrogen sulfide protects against lipopolysaccharide-induced acute lung injury in mice. Med Gas Res 2012, 2(1):26.

71. Xie K, et al: Molecular hydrogen ameliorates lipopolysaccharide-induced acute lung injury in mice through reducing inflammation and apoptosis. Shock 2012, 37(5):548-55.

72. Katakura $M$, et al: Hydrogen-rich water inhibits glucose and alpha,beta dicarbonyl compound-induced reactive oxygen species production in the SHR.Cg-Leprcp/NDmcr rat kidney. Med Gas Res 2012, 2(1):18.

73. Peters $\mathrm{O}$, et al: Increased formation of reactive oxygen species after permanent and reversible middle cerebral artery occlusion in the rat. J Cereb Blood Flow Metab 1998, 18(2):196-205.

74. Domoki F, et al: Hydrogen is neuroprotective and preserves cerebrovascular reactivity in asphyxiated newborn pigs. Pediatr Res 2010 68(5):387-92.

75. Ohno K, Ito M, Ichihara M: Molecular hydrogen as an emerging therapeutic medical gas for neurodegenerative and other diseases. Oxid Med Cell Longev 2012:353152. Epub.

76. Ghanizadeh A: Hydrogen as a novel hypothesized emerging treatment for oxidative stress in autism. Eur Rev Med Pharmacol Sci 2012, 16(9):1313-4.

77. Ghanizadeh A: Physical exercise and intermittent administration of lactulose may improve autism symptoms through hydrogen production. Med Gas Res 2012, 2(1):19.

78. Aoki K, et al: Pilot study: Effects of drinking hydrogen-rich water on muscle fatigue caused by acute exercise in elite athletes. Med Gas Res 2012, 2(1):12.

79. Nakao A, et al: Effectiveness of hydrogen rich water on antioxidant status of subjects with potential metabolic syndrome-an open label pilot study. J Clin Biochem Nutr 2010, 46(2):140-9.

80. Ito $\mathrm{M}$, et al: Drinking hydrogen water and intermittent hydrogen gas exposure, but not lactulose or continuous hydrogen gas exposure, 
prevent 6-hydorxydopamine-induced Parkinson's disease in rats. Med Gas Res 2012, 2(1):15.

81. Zhang J, et al: Effect of hydrogen gas on the survival rate of mice following global cerebral ischemia (Shock 37(6), 645-652, 2012). Shock 2012, 38(4):444.

82. Zhan $Y$, et al: Hydrogen gas ameliorates oxidative stress in early brain injury after subarachnoid hemorrhage in rats. Crit Care Med 2012, 40(4):1291-6.

83. Yoritaka A, et al: Pilot study of $\mathrm{H}(2)$ therapy in Parkinson's disease: a randomized double-blind placebo-controlled trial. Mov Disord 2013 [Epub ahead of print].

84. Shi P, Sun W: A hypothesis on chemical mechanism of the effect of hydrogen. Med Gas Res 2012, 2(1):17.

85. Hanaoka T, et al: Molecular hydrogen protects chondrocytes from oxidative stress and indirectly alters gene expressions through reducing peroxynitrite derived from nitric oxide. Med Gas Res 2011, 1(1):18.

86. Ishibashi T, et al: Consumption of water containing a high concentration of molecular hydrogen reduces oxidative stress and disease activity in patients with rheumatoid arthritis: an open-label pilot study. Med Gas Res 2012, 2(1):27.

87. Kang KM, et al: Effects of drinking hydrogen-rich water on the quality of life of patients treated with radiotherapy for liver tumors. Med Gas Res 2011, 1(1):11.

88. Ono $\mathrm{H}$, et al: Hydrogen $\left(\mathrm{H}_{2}\right)$ treatment for acute erythymatous skin diseases. A report of 4 patients with safety data and a non-controlled feasibility study with $\mathrm{H} 2$ concentration measurement on two volunteers. Med Gas Res 2012, 2(1):14.

89. Ito $\mathrm{M}$, et al: Open-label trial and randomized, double-blind, placebocontrolled, crossover trial of hydrogen-enriched water for mitochondrial and inflammatory myopathies. Med Gas Res 2011, 1(1):24.

90. Ohta S, Nakao A, Ohno K: The 2011 Medical Molecular Hydrogen Symposium: An inaugural symposium of the journal Medical Gas Research. Med Gas Res 2011, 1(1):10.

91. Ohta S: Recent progress toward hydrogen medicine: potential of molecular hydrogen for preventive and therapeutic applications. Curr Pharm Des 2011, 17(22):2241-52.

doi:10.1186/2045-9912-3-11

Cite this article as: Ghanizadeh and Berk: Molecular hydrogen: an overview of its neurobiological effects and therapeutic potential for bipolar disorder and schizophrenia. Medical Gas Research 2013 3:11.

\section{Submit your next manuscript to BioMed Central and take full advantage of:}

- Convenient online submission

- Thorough peer review

- No space constraints or color figure charges

- Immediate publication on acceptance

- Inclusion in PubMed, CAS, Scopus and Google Scholar

- Research which is freely available for redistribution 